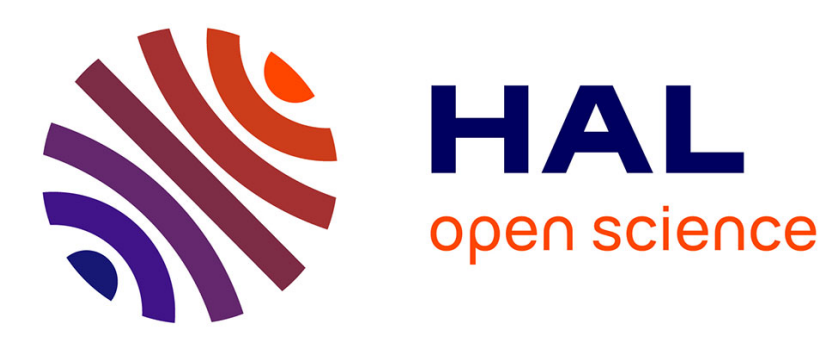

\title{
Competition for migrating customers: a game-theoretic analysis in a regulated regime
}

Patrick Maillé, Maurizio Naldi, Bruno Tuffin

\section{To cite this version:}

Patrick Maillé, Maurizio Naldi, Bruno Tuffin. Competition for migrating customers: a game-theoretic analysis in a regulated regime. Globecom'08 - IEEE Global Communication Conference, Sep 2008, New Orleans, United States. pp.1-5, 10.1109/GLOCOM.2008.ECP.316 . hal-02162365

\section{HAL Id: hal-02162365 \\ https://hal.science/hal-02162365}

Submitted on 21 Jun 2019

HAL is a multi-disciplinary open access archive for the deposit and dissemination of scientific research documents, whether they are published or not. The documents may come from teaching and research institutions in France or abroad, or from public or private research centers.
L'archive ouverte pluridisciplinaire HAL, est destinée au dépôt et à la diffusion de documents scientifiques de niveau recherche, publiés ou non, émanant des établissements d'enseignement et de recherche français ou étrangers, des laboratoires publics ou privés. 


\section{Competition for migrating customers: a game-theoretic analysis in a regulated regime}

\author{
Patrick Maillé \\ TELECOM Bretagne \\ 2, rue de la Châtaigneraie \\ CS 17607 \\ 35576 Cesson Sévigné Cedex, France \\ Email: patrick.maille@telecom-bretagne.eu
}

\author{
Maurizio Naldi \\ Università di Roma Tor Vergata \\ Dip. di Informatica Sistemi Produzione \\ Via del Politecnico 1 \\ 00133 Roma, Italy \\ Email: naldi@disp.uniroma2.it
}

\author{
Bruno Tuffin \\ INRIA Rennes - Bretagne Atlantique \\ Campus Universitaire de Beaulieu \\ 35042 Rennes Cedex, France \\ Email: Bruno.Tuffin@inria.fr
}

\begin{abstract}
Migration processes of customers between alternative providers are becoming more and more relevant. Providers competing for migrating customers may adopt a delaying strategy to retain customers who are willing to leave, facing regulatory sanctions for that unfair behaviour. A game-theoretic model is proposed to describe the resulting competition among providers. For that model, both stable and unstable Nash equilibria are shown to exist and the providers' equilibrium strategies can be derived, in general numerically. In the stable equilibrium case the delaying strategy predicted by the model introduces a mean delay that is a strongly nonlinear (decaying) function of the sanction value.
\end{abstract}

\section{INTRODUCTION}

The end of the monopolistic era in telecommunications services has spurred the entry of a number of competing providers in the market arena. Customers can now choose among many different offers for the same service and are allowed to freely migrate from a provider to another. Migration phenomena (often indicated as churn) are particulary relevant for mobile services, where annual churn rates as high as $25 \%$ are often observed [1] and studies have been devoted to ascertain the churn determinants (see e.g. the recent [2] and [3]). In any migration process we can identify four stakeholders: the customer (who initiates the process), the recipient provider, the losing provider, and the regulatory authority (which sets the rules for migration and checks for compliance). Since the customer's migration represents an economic loss for the losing provider, the latter has no interest in accelerating the migration process. Even when that process is led by the recipient provider, the losing provider has the possibility of delaying it, to the extent of reducing the effectiveness of the liberalization process [4]. In the fight for customer retention the losing provider may often be led to adopt unfair practices, calling for the intervention of the regulatory authority, e.g. through appropriate economic sanctions. The losing provider must therefore evaluate the convenience of its delaying strategy, by taking into account the pros linked to retaining the customer (continuation of the revenue stream associated to that customer) and the cons due to the possibility of incurring the sanctions for the undue delay in migration operations. The analysis reported in [5] has shown that the losing provider may identify a maximum tolerable sanction value, relating it to the customer lifetime value, i.e. the present value of the future revenue stream generated by the customer. Though the analysis has so far focused on the losing provider, the relative ease with which the customer may change his provider may however lead to a back-and-forth situation, in which competing providers play both roles. In fact a provider may be a losing one as well as a recipient one for a) the same customer at different times; b) different customers at the same time. The resulting competition between providers may be suitably modelled through a game, where each provider has to choose its delaying strategy. In this paper we propose a game-theoretic model with two providers, that takes into account the strategies of both providers competing for a migrating customer as well as the behaviour of the customer and the danger represented by sanctions. The model is then used to study the Nash equilibria of the interaction among providers, and analyze the influence of the sanction level on those equilibria.

\section{MARKOV CHAIN MODEL OF USERS' BEHAVIOR}

The goal of this section is to model the switching behavior of a customer between two available providers called A and B. We assume that it is represented by the Markov chain ${ }^{1}$ depicted in Figure 1, the meaning of the four states being defined in Table I. The transition from state 1 to state 2 (and

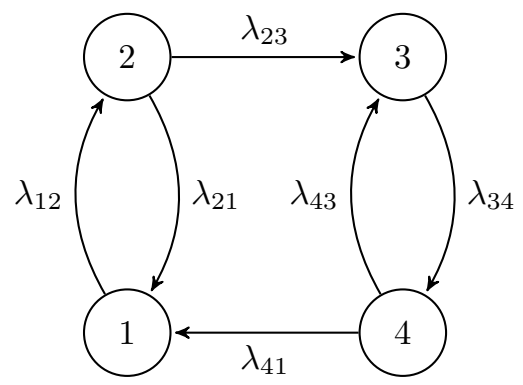

Figure 1. Markov chain model of the customer's switching behaviour

from state 3 to state 4 ) is for the customer willing to migrate

\footnotetext{
${ }^{1}$ We therefore implicitely assume that all events leading to a state change occur after an exponentially distributed time.
} 


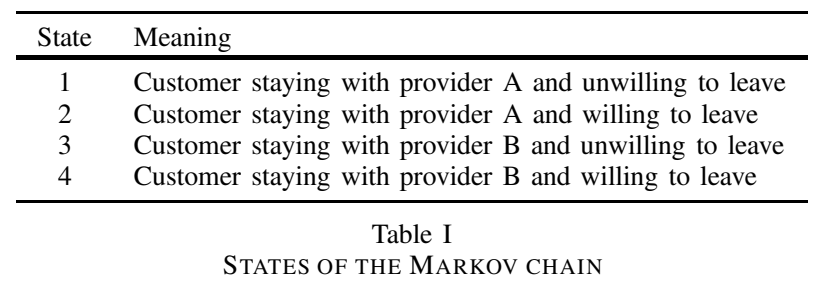

to the other provider; the reverse transitions model instead the customer reneging to the migration (due to winback actions by the losing provider or to its delaying strategies). The actual migration rates (from state 2 to state 3 , and from state 4 to state 1) depend either on a) the delay introduced by the losing provider in the porting operation, or on b) the impatience of the customer forcing the losing provider to comply with the migration-supporting regulations. The resulting infinitesimal generator is

$$
\mathbf{R}:=\left(\begin{array}{cccc}
-\lambda_{12} & \lambda_{12} & 0 & 0 \\
\lambda_{21} & -\left(\lambda_{21}+\lambda_{23}\right) & \lambda_{23} & 0 \\
0 & 0 & -\lambda_{34} & \lambda_{34} \\
\lambda_{41} & 0 & \lambda_{43} & -\left(\lambda_{41}+\lambda_{43}\right)
\end{array}\right) .
$$

From standard Markov chain analysis, the steady-state probability for each of the four states given by line vector $\pi=\left(\pi_{i}\right)_{i=1, \ldots, 4}$ exists $^{2}$ and is given by the solution of equations

$$
\pi R=0, \quad \sum_{i=1}^{4} \pi_{i}=1
$$

If $c:=\lambda_{34} \lambda_{41}\left(\lambda_{23}+\lambda_{21}\right)+\lambda_{12} \lambda_{23}\left(\lambda_{41}+\lambda_{43}\right)+\lambda_{12} \lambda_{34}\left(\lambda_{41}+\lambda_{23}\right)$, it can be readily checked that the solution is

$$
\begin{aligned}
\pi_{1} & =\frac{\lambda_{34} \lambda_{41}\left(\lambda_{23}+\lambda_{21}\right)}{c} \\
\pi_{2} & =\frac{\lambda_{34} \lambda_{41} \lambda_{12}}{c} \\
\pi_{3} & =\frac{\lambda_{12} \lambda_{23}\left(\lambda_{41}+\lambda_{43}\right)}{c} \\
\pi_{4} & =\frac{\lambda_{12} \lambda_{23} \lambda_{34}}{c} .
\end{aligned}
$$

The question is now, how do we relate some of the transition rates appearing in the infinitesimal generator to the relevant parameters of the problem? Our goal being to study the retention policy of providers and defining regulation rules, we introduce the average delay imposed by the two providers, respectively $T_{A}$ and $T_{B}$, and the suing rate $\mu$ taking into account the attitude of the customer to force the migration by taking legal actions against the delaying provider. We then express the transition rates that mark the migration to the other provider, i.e. $\lambda_{23}$ and $\lambda_{41}$, as the sum of two rates, since either

\footnotetext{
${ }^{2}$ Remark that when a provider $i$ sets $T_{i}=0$, the Markov chain described before is degenerate: if $T_{A}=0$ for example then states 2 and 3 are only one state. However the corresponding Markov chain with fewer states remains ergodic as soon as all transmission rates are strictly positive, which will be the case in the examples we consider.
}

the end of the retention period or the legal action taken by the customer leads to a provider change:

$$
\begin{aligned}
& \lambda_{23}=\frac{1}{T_{A}}+\mu, \\
& \lambda_{41}=\frac{1}{T_{B}}+\mu .
\end{aligned}
$$

With full generality, it seems also relevant that the other rates depend on $T_{A}$ and $T_{B}$ too. Indeed, a customer could be more inclined to switch (and to renege) depending on the respective retention times of providers. We therefore let them depend on the retention times, i.e., $\lambda_{12}=\lambda_{12}\left(T_{A}, T_{B}\right), \lambda_{21}=$ $\lambda_{21}\left(T_{A}, T_{B}\right), \lambda_{34}=\lambda_{34}\left(T_{A}, T_{B}\right)$ and $\lambda_{43}=\lambda_{43}\left(T_{A}, T_{B}\right)$. Note that other parameters such as reputation of the provider, price, etc., can also be included in those rates. Though, not being the purpose of the current study, they are just hidden and kept constant.

\section{NON-COOPERATIVE GAME DESCRIPTION AND ALGORITHMIC SOLUTION}

The customer behavior having been described, we can now investigate provider strategies. providers have an open interest in keeping the customer, since any delay in the migration allows the losing provider to keep the revenues associated to that customer. On the other hand the legal actions taken by the customer lead to a sanction $s_{0}$. The utility function of each provider, representing here their financial net benefits per time unit at steady-state, is therefore given by the difference between the average net profits associated to the customer (respectively net revenues associated to customers $p_{A}$ or $p_{B}$ times the probability of keeping the customer) and the average sanction (which is levied only if the customer has expressed his intention to leave the provider and has taken a legal action). The resulting expressions of the utility function for the two providers are therefore

$$
\begin{aligned}
& U_{A}=p_{A}\left(\pi_{1}+\pi_{2}\right)-s_{0} \pi_{2} \mu, \\
& U_{B}=p_{B}\left(\pi_{3}+\pi_{4}\right)-s_{0} \pi_{4} \mu .
\end{aligned}
$$

In this competitive environment, each provider strives to find its best strategy, i.e., its average retention time (assuming here fixed revenues) maximizing its utility. But its utility depends not only upon its own choice (from values of steady-state probabilities $\left.\pi_{i}, i \in\{1, \ldots, 4\}\right)$, but also upon the strategy choice of the opponent provider. In this situation, the solution concept is that of Nash equilibrium from non-cooperative game theory [6].

A Nash equilibrium is here an average retention time profile $T^{*}=\left(T_{A}^{*}, T_{B}^{*}\right)$ from which no provider has any incentive to deviate unilaterally. Formally,

$$
\begin{aligned}
& T_{A}^{*} \in \operatorname{argmax}_{T_{A} \in \mathcal{S}} U_{A}\left(T_{A}, T_{B}^{*}\right) \text { and } \\
& T_{B}^{*} \in \operatorname{argmax}_{T_{B} \in \mathcal{S}} U_{B}\left(T_{A}^{*}, T_{B}\right),
\end{aligned}
$$

i.e., the best strategy provider $i \in\{A, B\}$ can use is $T_{i}^{*}$ given that the strategy of the other provider is $T_{j}^{*}$ (with $j \in\{A, B\}$, $j \neq i$ ). In (4), $\mathcal{S}$ is the strategy set of each provider. In general we search our solution in the set of non-negative real numbers 
$\mathcal{S}:=\mathbb{R}_{+}$, however we consider that very large values of the retention time (larger than 1 year for example) are not realistic and should not be considered. For the numerical analysis carried out in Section $\mathrm{V}$, we therefore take $\mathcal{S}=\left[O, T_{\max }\right]$ for a given value of $T_{\max }$, which makes the strategy set of each provider a compact and bounded set.

To determine practically the Nash equilibria (if any) of the game played among providers, we define the best response of each provider as a function $\mathcal{S} \mapsto 2^{\mathcal{S}}$ of the strategy of its opponent. Those best response functions are

$$
\begin{aligned}
& \operatorname{BR}_{A}\left(T_{B}\right):=\arg \max _{T_{A} \in \mathcal{S}} U_{A}\left(T_{A}, T_{B}\right) \text { and } \\
& \operatorname{BR}_{B}\left(T_{A}\right):=\arg \max _{T_{B} \in \mathcal{S}} U_{B}\left(T_{A}, T_{B}\right) .
\end{aligned}
$$

If we define the best response correspondance $\overline{\mathrm{BR}}: \mathcal{S} \times \mathcal{S} \mapsto$ $2^{\mathcal{S}} \times 2^{\mathcal{S}}$ as $\overline{\mathrm{BR}}\left(T_{A}, T_{B}\right):=\left\{\left(t_{A}, t_{B}\right) \in \mathcal{S} \times \mathcal{S}: t_{A} \in\right.$ $\left.\mathrm{BR}_{A}\left(T_{B}\right), t_{B} \in \mathrm{BR}_{B}\left(T_{A}\right)\right\}$, then a Nash equilibrium is simply a fixed point of the 2-dimensional function $\overline{\mathrm{BR}}$.

Therefore an exhaustive way to proceed to find Nash equilibria is to follow Algorithm 1 described below.

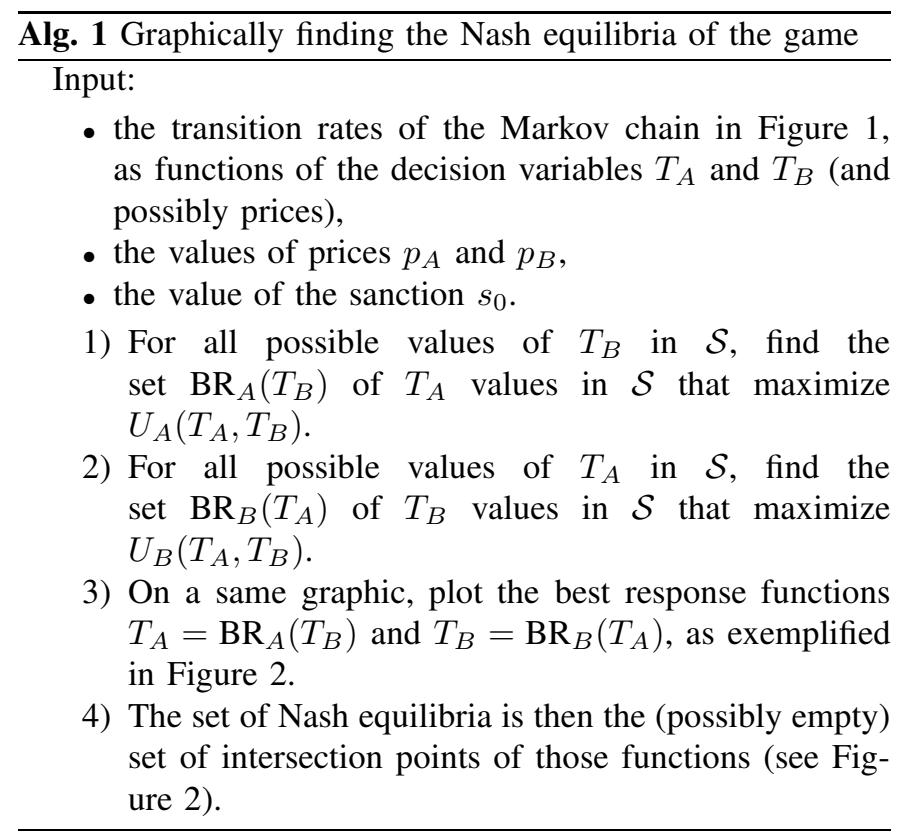

Note that when the analytical derivation of the best response is not feasible in step 1 of the algorithm, only a finite number of values can be tried in practice. In the numerical results presented in Section V, we use a given number (say, 500) of equally spaced values in $\mathcal{S}$.

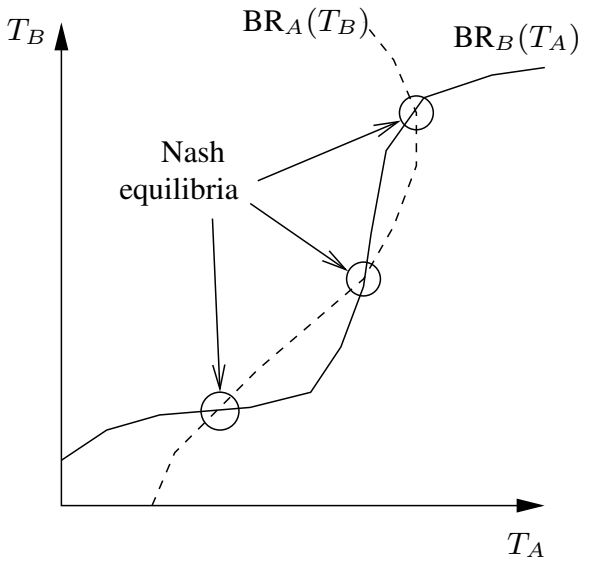

Figure 2. Graphical determination of Nash equilibria (here the best response of each user is unique, but this need not be the case in general).

\section{ANALYSIS OF THE GAME IN A SIMPLIFIED SETTING}

Consider the special case where

$$
\begin{aligned}
\lambda_{12} & =\lambda \\
\lambda_{34} & =\lambda+\nu \\
\lambda_{21} & =\alpha \\
\lambda_{43} & =\alpha \\
\lambda_{23} & =\frac{1}{T_{A}}+\mu \\
\lambda_{41} & =\frac{1}{T_{B}}+\mu,
\end{aligned}
$$

for some fixed nonnegative values $\lambda, \nu, \alpha$, and $\mu$. That is, only the migration rates depend on retention times: the willingness to leave or the reneging behavior are assumed constant. We take $\lambda_{34}>\lambda_{12}$ to introduce some asymetry into the game; this models the fact that provider A has for instance a better reputation (or could provide a better quality of service) than provider B. To avoid dealing with too many parameters and separating in several different cases, consider an arbitrarily chosen example where $\lambda=\nu=1, \alpha=2, \mu=4$ and $p_{A}=$ $p_{B}=1$. After simple computations, we get

$$
\begin{aligned}
U_{A} & =2 \frac{\left(1+4 T_{B}\right)\left(1+7 T_{A}-4 s_{0} T_{A}\right)}{3+18 T_{A}+16 T_{B}+88 T_{B} T_{A}} \\
U_{B}= & \frac{\left(1+4 T_{A}\right)\left(1+8 T_{B}-8 s_{0} T_{B}\right)}{3+18 T_{A}+16 T_{B}+88 T_{B} T_{A}} .
\end{aligned}
$$

We now consider the Nash equilibria of the game. Since we manage to carry out an analytical study, we can take $\mathcal{S}=\mathbb{R}_{+}$ here. Computing the partial derivatives of the utility functions gives

$$
\begin{aligned}
& \frac{\partial U_{A}}{\partial T_{A}}=2 \frac{\left(1+4 T_{B}\right)\left(3-12 s_{0}+8\left(3-8 s_{0}\right) T_{B}\right)}{\left(3+18 T_{A}+16 T_{B}+88 T_{B} T_{A}\right)^{2}} \\
& \frac{\partial U_{B}}{\partial T_{B}}=8 \frac{\left(1+4 T_{A}\right)\left(1-3 s_{0}+\left(7-18 s_{0}\right) T_{A}\right)}{\left(3+18 T_{A}+16 T_{B}+88 T_{B} T_{A}\right)^{2}} .
\end{aligned}
$$

We remark here that the numerator of each derivative does not depend on the average retention time of the corresponding 
provider. It can actually be easily checked that whatever the values of parameters $\lambda, \nu, \alpha, \mu, p_{A}$ and $p_{B}$, this property is verified.

Note also that $\frac{\partial U_{A}}{\partial T_{A}}$ can be zero iff $1 / 4 \leq s_{0} \leq 3 / 8$. Below, it is always positive and above, always negative. Same thing for $\frac{\partial U_{A}}{\partial T_{A}}$ which can be zero iff $1 / 3 \leq s_{0} \leq 7 / 18$.

Depending on the value of $s_{0}$, we end up with several possible cases.

- If $s_{0} \leq 1 / 3$, provider B's only interest is to set $T_{B}=\infty$, and therefore, the same strategy applies for provider A. The unique Nash equilibrium is then $(\infty, \infty)$. The reason is that the sanction is too low to prevent the providers from retaining abusively the customers.

- Similarly, if $s_{0} \geq 3 / 8$, provider A's interest is to set $T_{A}=0$. At this value, the derivative of $U_{B}$ with respect to $T_{B}$ is negative, meaning that $T_{B}=0$ is the best response. The resulting unique Nash equilibrium is $(0,0)$. Here, the sanction is too high for providers, their interest is to let customers leave if they are willing to.

- Now, if $1 / 3<s_{0}<3 / 8$, we then end up with three possible Nash equilibria: $(0,0),(\infty, \infty)$ and $\left(T_{A}^{*}, T_{B}^{*}\right)=$ $\left(\frac{1-3 s_{0}}{7-18 s_{0}}, \frac{3-12 s_{0}}{8\left(3-8 s_{0}\right)}\right)$. Indeed, in the later case, both derivatives are null and we are at a (individual) maximum for each provider. On the other hand, as soon as a player $i \in\{A, B\}$ plays $T_{i}>T_{i}^{*}$, the other player has a maximum at $\infty$, and then $i$ also. The reasoning is similar for $T_{i}<T_{i}^{*}$, leading to $(0,0)$.

This illustrates the interest of the analysis: determining threshold values on the sanction fee in order to prevent providers from retaining customers.

\section{NUMERICAL ANALYSIS IN A COMPLEX CASE}

In this section, we consider a more complex setting, where all transition rates depend on the relative quality of the two providers (via their mean retention time $T$ ). We use here the following model:

$$
\begin{aligned}
& \lambda_{12}=\lambda \cdot\left(1-\omega+\omega \frac{T_{B}-T_{A}}{T_{A}+T_{B}}\right) \\
& \lambda_{34}=\lambda \cdot\left(1-\omega+\omega \frac{T_{A}-T_{B}}{T_{A}+T_{B}}\right)+\nu \\
& \lambda_{21}=\alpha \cdot\left(1-\omega+\omega \frac{T_{A}-T_{B}}{T_{A}+T_{B}}\right) \\
& \lambda_{43}=\alpha \cdot\left(1-\omega+\omega \frac{T_{B}-T_{A}}{T_{A}+T_{B}}\right) \\
& \lambda_{23}=\frac{1}{T_{A}}+\mu \\
& \lambda_{41}=\frac{1}{T_{B}}+\mu,
\end{aligned}
$$

where $\lambda, \nu, \alpha$ and $\mu$ are constant as in the previous section, and $\omega$ is a constant that represents users sensitivity to providers reputation in their churning decisions. This model reflects the fact that a user is less likely to leave a provider that retains customers, and is also more likely to renege because he is reluctant to have to fight to churn. With such a model, providers have an even stronger incentive to use retention policies, because of the direct gain in terms of income during the retention period and the indirect gain due to the fact that users are less willing to leave.

Since an analytical study like the one carried out in the previous section is not realizable anymore, we use numerical computation following Algorithm 1 to determine the Nash equilibria of the game and the influence of sanctions. We present next an example where time is expressed in years, with $\lambda=1 / 5$ (which corresponds to around $20 \%$ of customers willing to churn each year if $\left.T_{A}=T_{B}\right), \nu=1 / 10, \alpha=1 / 6$ (mean reneging time of 2 months) and $\mu=1 / 6$. We also take $T_{\max }=1$ year, i.e. $\mathcal{S}=[0,1]$, and $p_{A}=p_{B}=p$ (no price game among providers). Remark that due to the utility functions (3), when both providers get the same revenues $p$ then the outcomes of the game depend only on the ratio $s_{0} / p$.

Without sanctions $\left(s_{0}=0\right)$, our numerical computations highlight that best response functions $\mathrm{BR}_{A}$ and $\mathrm{BR}_{B}$ simply consist in choosing the highest possible retention time, i.e. $\forall T \in \mathcal{S}, \mathrm{BR}_{A}(T)=\mathrm{BR}_{B}(T)=T_{\text {max }}$. Therefore a sanction $s_{0}$ needs to be introduced to incentivize providers to reduce their retention times. Figure 3 plots the best response functions of both providers in the case when $s_{0}=10 p$. It appears that

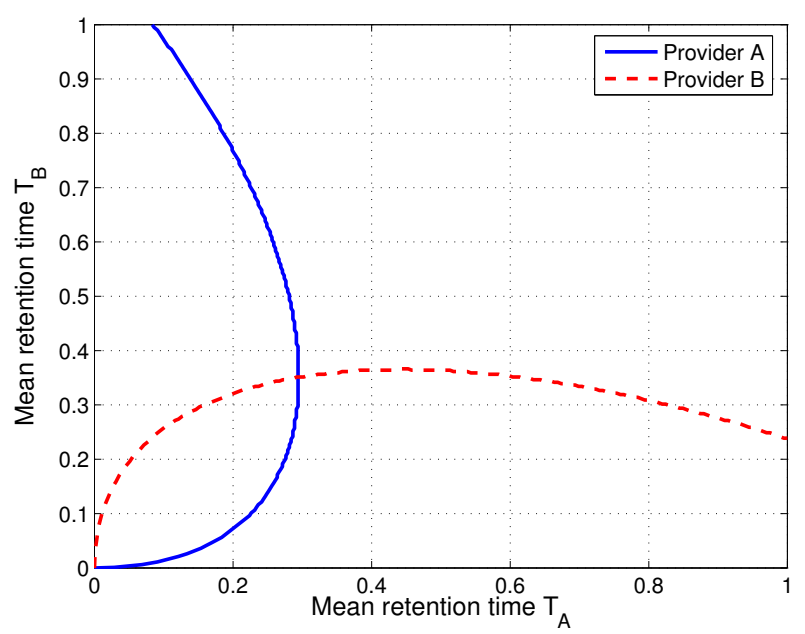

Figure 3. Nash equilibria with sanction $s_{0}=10 p$

there are two Nash equilibria, namely $\left(T_{A}, T_{B}\right)=(0,0)$ and $\left(T_{A}, T_{B}\right)=(0.29,0.35)$. Notice however that only the latter equilibrium is stable, since a small deviation of any of the two providers from the point $\left(T_{A}, T_{B}\right)=(0,0)$ leads the best response dynamics to that equilibrium. Notice also that at this equilibrium, the provider that benefits from a better reputation (modelled by the $\nu$ parameter) retains his customers less than its opponent.

Increasing more the sanction value gives the same form of best response functions as those presented in Figure 3. We therefore focus now on the stable Nash equilibrium that a given sanction level yields.

Figure 4 plots the strategies of each provider predicted by 
our model when the sanction level $s_{0} / p$ changes. As could be

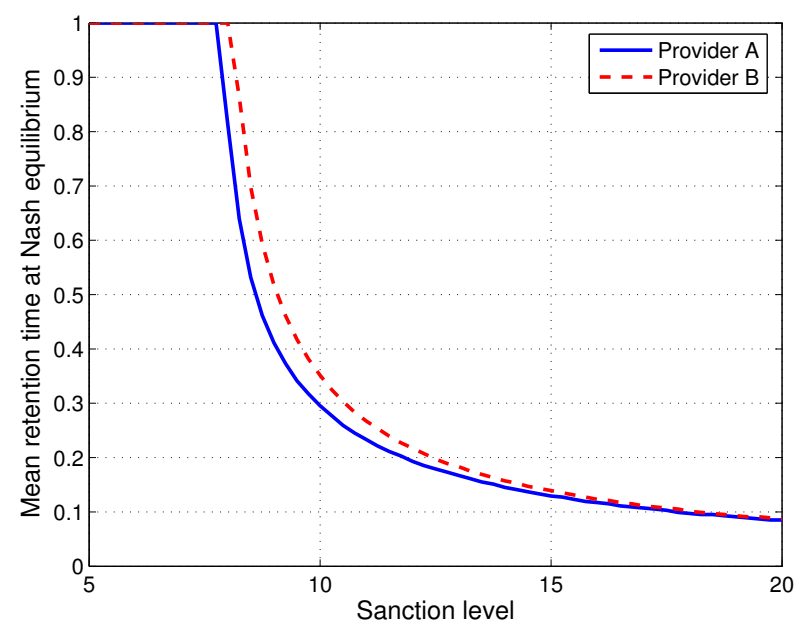

Figure 4. Stable Nash equilibrium strategies when the sanction varies.

expected, increasing the sanction level indeed makes providers reduce their mean retention time. However, unlike what was observed in the simplified model of the previous section, it seems that there is no threshold here for the value of $s_{0}$ above which the only Nash equilibrium is $(0,0)$. Therefore the sanction level has to be chosen such that the resulting retention times be sufficiently small (say, less than 0.1 year, which from Figure 4 corresponds to $s_{0} \geq 18 p$ ).

To compare the providers' perception of both Nash equilibria of the game, we plot in Figure 5 their utilities at those two possible outcomes when the sanction $s_{0} / p$ varies. Notice

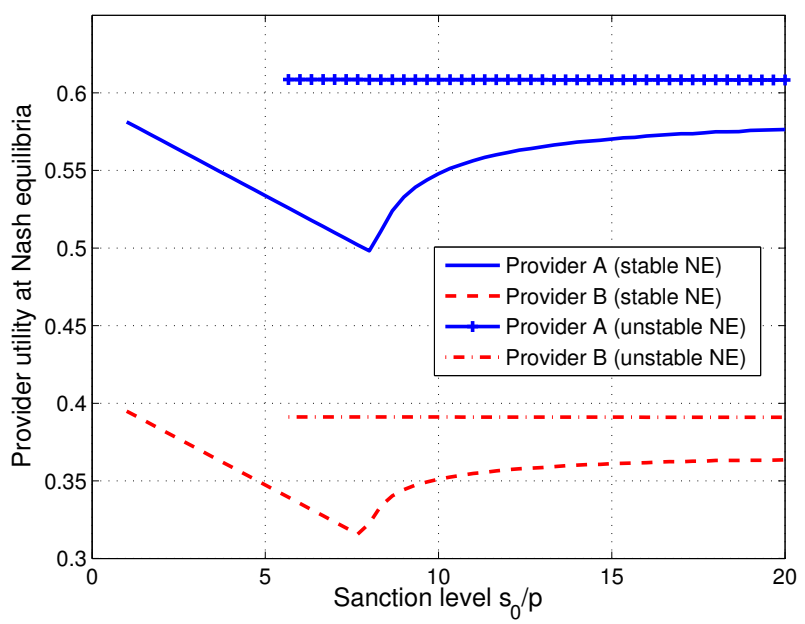

Figure 5. Providers' utility at stable and unstable (if any) Nash equilibria.

that $\left(T_{A}, T_{B}\right)=(0,0)$ is not a Nash equilibrium when the sanction is below $5.6 p$. In that case, the game has only one Nash equilibrium (that is stable). This equilibrium consists in each provider setting the largest possible retention time (here
$T_{\max }=1$ ). We observe that provider A obtains a larger utility than provider B in all cases due to its advantage $\nu$.

Interestingly, it appears that both players would be better off playing the unstable Nash equilibrium instead of the stable one. Therefore the use of the sanction is justified by its effect on the stable equilibrium: enforcing users to reduce their retention time makes the outcome $\left(T_{A}, T_{B}\right)$ get closer to the more efficient situation $(0,0)$. Moreover, we also notice in Figure 5 that when the stable equilibrium is different from $\left(T_{\max }, T_{\max }\right)$, i.e. for $s_{0} / p \geq 8$ in our example, an increase in the sanction unexpectedly leads to an increase in both providers' utility. This also justifies the use of the sanction. Consequently, we can say that for this model, the sanction improves the user perceived quality of both providers (that is decreasing in their retention times), but can also be beneficial to both providers by enforcing their opponent to reduce its retention time.

\section{CONCLUSIONS}

A game-theoretic model has been developed to describe the behaviour of two service providers competing for migrating customers. The model takes into account all the stakeholders, namely the delaying behaviour of the two providers, the sanctions levied by the regulatory authorities, and the impatience of the customer waiting for the completion of the migration process. A simplified setting has been examined to show the use of the model. In that setting it has been shown that both stable and unstable Nash equilibria exist. The stable equilibrium retention strategies, consisting in determining appropriate mean delaying times, have been derived for both providers. The game outcome predicted mean delay of each provider appears to be a strongly non linear function of the sanction imposed by the regulator.

\section{ACKNOWLEDGMENT}

The authors would like to acknowledge the support of Euro-FGI Network of Excellence through the specific research project CAP (Competition Among Providers).

\section{REFERENCES}

[1] K. Wieland, "The customer retention challenge," Telecommunications, vol. 40, no. 10, pp. 14-17, October 2006.

[2] J. Ahn, S. Han, and Y. Lee, "Customer churn analysis: Churn determinants and mediation effects of partial defection in the korean mobile telecommunications service industry," Telecommunications Policy, vol. 30, no. 10-11, pp. 552-568, November-December 2006.

[3] A. Eshghi, D. Haughton, and H. Topi, "Determinants of customer loyalty in the wireless telecommunications industry," Telecommunications Policy, vol. 31, no. 2, pp. 93-106, March 2007.

[4] S. Buehler, R. Dewenter, and J. Haucap, "Mobile number portability in Europe," Telecommunications Policy, vol. 30, no. 7, pp. 385-399, August 2006.

[5] M. Naldi, "A simple model for the effectiveness of delaying strategies for telecommunications churn reduction," in 10th International Conference on Computer Modelling and Simulation EUROSIM 2008, Cambridge, 13 April 2008.

[6] M. Osborne and A. Rubinstein, A Course on Game Theory. MIT Press, 1994. 